\title{
Piezoelectrically Driven Silicon Beam Force Sensor
}

\author{
C. J. VAN MULLEM, F. R. BLOM*, J. H. J. FLUITMAN and M. ELWENSPOEK
}

MESA, Institute for Micro Electronics, Materials Engineering, Sensors and Actuators, University of Twente, P.O. Box 217, 7500 AE Enschede (The Netherlands)

\begin{abstract}
A resonant silicon beam force sensor with piezoelectric excitation and detection is being developed. The realization is based on IC and thin-film technology with $\mathrm{ZnO}$ as the piezoelectrical layer. The theory, realization and measurements of a bent-frame sensors are described. A frequency shift of about 3.3 times the unloaded resonance frequency $f_{0}$ $\left(f_{0} \simeq 6 \mathrm{kHz}\right)$ is measured with an external load force up to $0.4 \mathrm{~N}$. The absolute sensitivity of the force sensor is $64 \mathrm{kHz} / \mathrm{N}$ and the full-scale sensitivity is $29 \mathrm{kHz} / \mathrm{N}$. Using a simple model for the load-force transduction from external to sensor force, the measurements are in good agreement with the theory.
\end{abstract}

\section{Introduction}

Since 1983, research has been carried out at the University of Twente on resonant silicon structures [1]. One application of these structures is the resonant beam force sensor. This sensor can be used as a resonant strain gauge integrated in micromechanical sensors [2] or in electronic precision microscales, usually with a range of up to $1 \mathrm{~N}$ without forcereduction mechanisms and a resolution of 100 ppm or better.

The complete sensor design is shown in Fig. 1. In order to ensure safe manipulation, the sensor beam is supported by two bars of the original wafer thickness. On the sensor beam a multilayer structure is realized for the

\footnotetext{
*Present address, Oce, P.O. Box 101, 5900 MA Venlo, The Netherlands.
} $0924-4247 / 91 / \$ 3.50$ electrical excitation and detection of the vibration of the sensor beam using the piezoelectrical effect of a $\mathrm{ZnO}$ layer $[1,3]$. The bottom electrode is a highly boron doped $\left(\mathrm{p}^{++}\right)$) silicon layer and the top one is an aluminium layer.

In order to improve the efficiency of the electromechanical transduction of the $\mathrm{ZnO}$ at low frequencies, a $\mathrm{p}^{++}-\mathrm{ZnO}$ structure is used. This structure will have a better efficiency than the $\mathrm{p}^{++}-\mathrm{SiO}_{2}-\mathrm{ZnO}$ (MOS) structure presented in ref. 4 because of the complete depletion of the $\mathrm{ZnO}$ layer [5] and the absence of a voltage drop across the oxide layer in the MOS structure.

Measurements on a bare prototype using acoustic excitation and optical detection have already been reported [6]. In this paper the force sensor with piezoelectric excitation and detection is presented. This force sensor will be an integral part of the frequency-determining block of an electronic oscillator (Fig. 2). The output signal is then the frequency of an electrical signal where the oscillation frequency is the same as the resonance frequency. The next step in the development of the resonant force sensor will be the on-chip realization of the feedback circuit to create an integrated sensor [7].

\section{Force-to-frequency Conversion}

For small pulling forces $(F)$ the force-toresponse frequency conversion for a clamped beam can be approximated by [6]

$$
f \simeq f_{0}\left(1+0.295\left(\frac{l}{d}\right)^{2} \frac{F}{E A}+\beta F^{2}\right)^{1 / 2}
$$

(C) Elsevier Sequoia/Printed in The Netherlands 


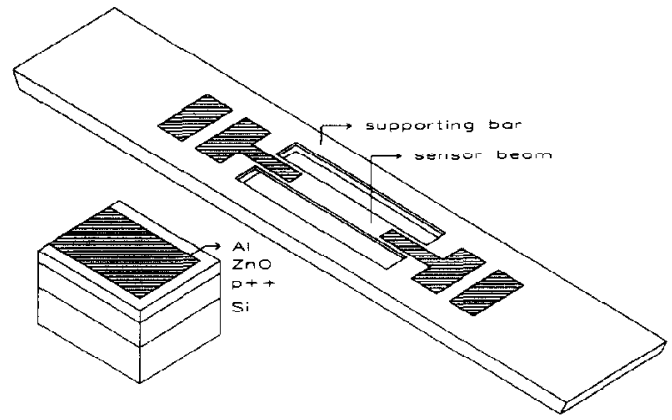

Fig. 1. Layout of the resonant force sensor with a close-up of the multilayer structure on the sensor beam.

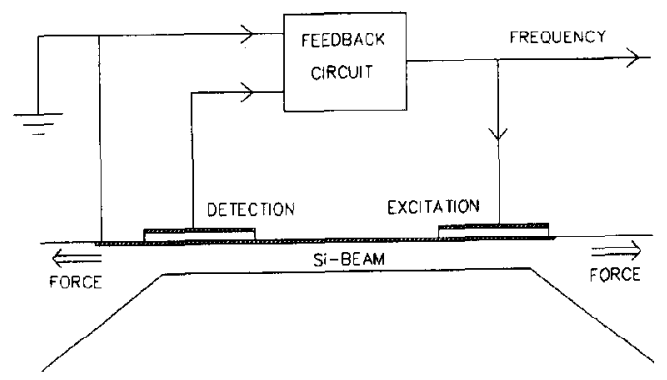

Fig. 2. Schematic of the silicon beam oscillator.

with $f$ the loaded and $f_{0}$ the unloaded resonance frequency, $E$ the Young's modulus of silicon, $l$ the length, $d$ the thickness, $A$ the cross section of the sensor beam and $\beta$ a parameter fitted with the exact conversion. The second-order term is included to obtain a more accurate approximation in the measured force range.

The realized force sensors contain a multilayer structure for excitation and detection. Due to the fabrication method of this composite structure, residual stresses occur in the beam. The tensile stress of the highly doped $\mathrm{Si}$ layer will compensate the compressive stress in the $\mathrm{ZnO}$ layer. In order to model the resulting stress, an off-set force $F_{\mathrm{r}}$ is accounted for in the force-to-frequency conversion, eqn. (1). The pulling force $F$ is replaced by $F_{\mathrm{r}}$ plus the actual pulling force $F_{\mathrm{s}}$ on the sensor beam. This off-set force determines the operation point of the force sensor, and for small forces the sensor has the highest sensitivity. The off-set force can be tailored by choosing the proper thickness of the various layers in the multilayer structure.

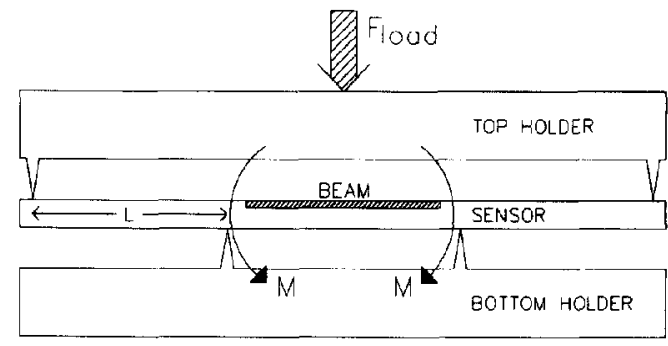

Fig. 3. The measurement set-up for the bent-frame sensor.

\section{Force Conversion of the Bent-frame Sensor}

There are several ways to load the force sensor with an external load. We chose a bent frame for this purpose. The principal set-up for this bent-frame sensor is shown in Fig. 3. The sensor is supported by two sharp ridges of the bottom sample holder. The distance between these two inner ridges is larger than the length $l$ of the sensor beam. On top of the sensor a second holder is placed which also has two ridges. This construction allows accurate force conversion up to a load of $0.4 \mathrm{~N}$.

The external load force $F_{\text {load }}$ is transformed by the frame into the axial force $F_{\mathrm{s}}$ on the sensor beam. The force conversion is given by [6]

$F_{\mathrm{s}}=3 L \frac{h b d}{b_{\mathrm{b}} d_{\mathrm{b}}^{3}} F_{\text {load }}-\frac{3}{8} L^{2} \frac{b d}{E}\left(\frac{l}{b_{\mathrm{b}} d_{\mathrm{b}}^{3}}\right)^{2} F_{\text {load }}^{2}$

with $L$ the distance between the outer and inner ridges of the sample holders, $h$ the distance between the neutral axis of the frame and the sensor beam, $b$ the width of the sensor beam, $b_{\mathrm{b}}$ the mean width and $d_{\mathrm{b}}$ the thickness of the supporting bars. The theoretical curve for this force conversion is given in Fig. 4.

The external load $F_{\text {load }}$ causes a constant bending moment $M$ between the inner ridges. This bending moment results in two displacements of the sensor beam. First, the sensor beam will be stretched because it lies above the neutral axis. The other displacement causes compression of the sensor beam because the end points of the supporting bars 


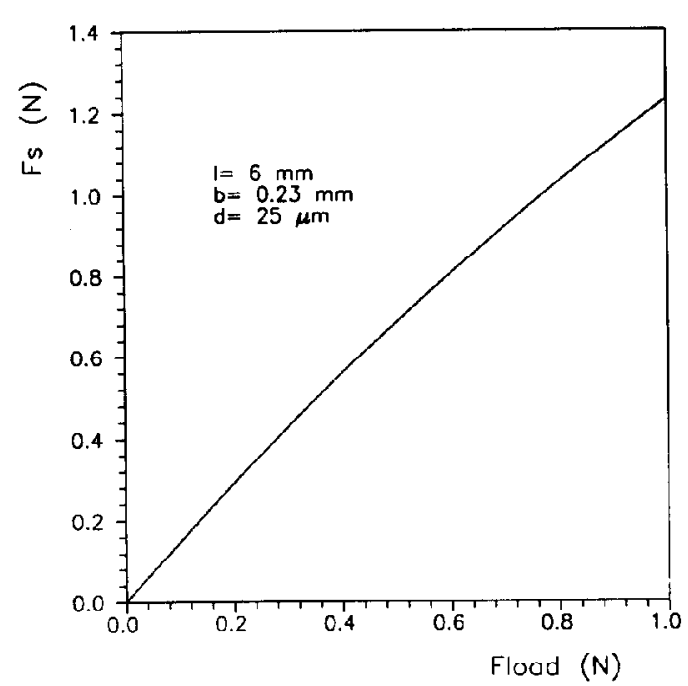

Fig. 4. Theoretical curve for the force conversion: $F_{\text {load }}$ vs. $F_{\mathrm{s}}$.

move closer to each other. The first displacement is proportional to $F_{\text {load }}$ and the second one is negative and proportional to the square of $F_{\text {load }}$. The deformation of the beam near the clamping can be neglected. The total displacement is a boundary condition for the sensor beam and is counteracted by a force $F_{\mathrm{s}}$ in the beam, resulting in eqn. (2).

The parameters which determine the force conversion can be optimized for maximum frequency shift in the working force range of the sensor. Hence, from this force conversion the design geometry of the bent-frame force sensor can be obtained.

\section{Realization}

The realization process of the force sensor can be divided into two stages: first, processing the front side of the wafer, depositing and patterning the layers on the beam and secondly, micromachining of the wafer using wet chemical etching of the reverse side and dry reactive ion etching (RIE) of the front side. The complete process comprises six photolithographic steps.

The starting material is a double-sided polished 2" (100) silicon wafer. Each wafer contains 12 force sensors. First the wafers are steam oxidized; the $\mathrm{SiO}_{2}$ layer is used as mask material for the bottom electrode and for the wet chemical etching of silicon later on in the process. The next step is the deposition of a boron glass layer by APCVD, the drive-in of the boron and the removal of the glass layer. A second $\mathrm{SiO}_{2}$ layer is grown to insulate the top from the bottom electrode.

The next step in the process is the sputtering of $\mathrm{ZnO}$. The etching of $\mathrm{ZnO}$ is performed in a combined $\mathrm{HAc}, \mathrm{H}_{3} \mathrm{PO}_{4}$ and $\mathrm{H}_{2} \mathrm{O}$ solution. After patterning the $\mathrm{ZnO}$, the aluminium top electrode is deposited by evaporation. A lift-off process is used to pattern the aluminium. After completing the front side processing, electrical measurements are performed to characterize the electrical properties of the multilayer structure.

The next step is the processing of the reverse side of the wafer to etch membranes which have the thickness of the sensor beam and $\mathrm{V}$-grooves between the different sensors in order to simplify the separation of the completed sensors. The anisotropic etching of silicon is performed in a $\mathrm{KOH}$ and $\mathrm{H}_{2} \mathrm{O}$ solution ( $1: 2$ by weight) at $75^{\circ} \mathrm{C}$. During this etch step the front side of the wafer is protected by using a Teflon holder.

The last step of the realization process is the dry etching of the silicon membranes from the front side in beams by RIE. Standard photoresist, which is easily removed in an oxygen plasma, can be used as mask material at this stage. The 12 sensors are separated by breaking along the $\mathrm{V}$-grooves.

\section{Measurements and Discussion}

In the measurement set-up the sensor is placed between the two holders. In order to make electrical contact with the sensor, aluminium wires are bonded between the four bond paths of the sensor and a special frame. These bonds are realized with an oversized wire length to ensure that they will not influence the $F_{\text {load }}$ to $F_{\mathrm{s}}$ force conversion. The external load can be applied by a mass placed on the top holder or by a fixed displacement 


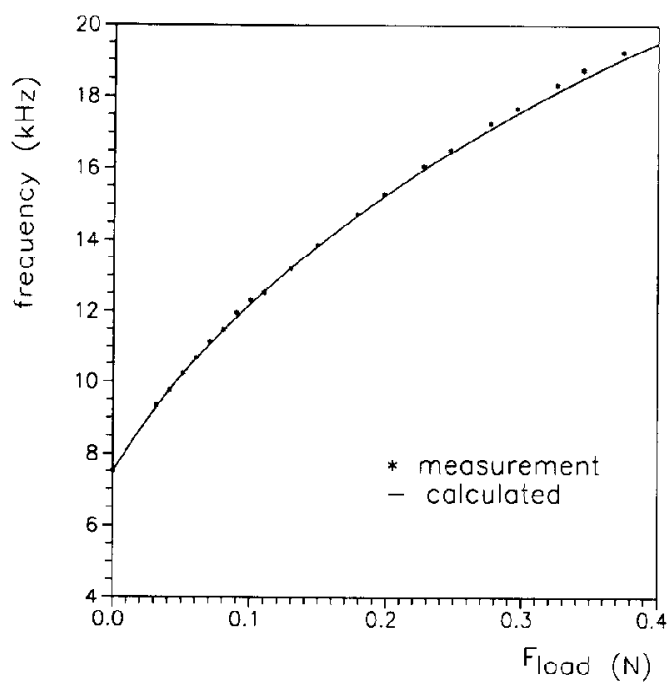

Fig. 5. Measured and calculated results: the resonance frequency vs. the external applied load force, $l=6 \mathrm{~mm}$, $b=0.23 \mathrm{~mm}, d=25 \mu \mathrm{m}$.

of the top holder. The electrical transfer of the sensor is measured with a gain/phase analyser to determine the resonance frequency as a function of the external load.

In Fig. 5 the measured and calculated conversion of the external force to resonance frequency is given. The unloaded resonance frequency is shifted due to a residual tensile stress in the multilayer structure from which an off-set force of $0.03 \mathrm{~N}$ can be calculated. The quality factor $Q$ is about 400 . The calculated curve includes the force conversion from $F_{\text {load }}$ to $F_{\mathrm{s}}$ and the force-to-frequency conversion. For larger forces the calculated curve starts to deviate more and more from the measured one. This may be caused by inaccuracy of the sensor dimensions and also second-order effects, like misalignment within the measurement set-up.

The maximum load that can be applied for the described devices is more than $0.8 \mathrm{~N}$. We found that it depends on the etch process used for the realization of the sensor beam. Bare prototypes were also realized where the front side was wet chemical etched instead of dry etched. The maximum load in this case was limited to $0.3 \mathrm{~N}$. If the maximum load was exceeded, the bare sensors broke in one of the corners of the supporting bars with the frame. Etching both sides anisotropically will result in sharp corners with high stress concentrations. This could be the explanation for the difference in the maximum applicable load.

In order to characterize the performance of the force sensor, the absolute and full-scale sensitivities are measured. The definition of the absolute sensitivity $S_{\mathrm{A}}$ is given by $\delta f / \delta F$ at $F=0 \mathrm{~N}$ and the full-scale sensitivity $S_{\mathrm{FS}}$ is defined by $\Delta f_{\mathrm{FS}} / \Delta F_{\mathrm{FS}}$, where $\Delta f_{\mathrm{FS}}$ is the total frequency swing occurring over the full-force range $\Delta F_{\mathrm{FS}}$. If the sensor behaves linearly over the full-force range $\Delta F_{\mathrm{FS}}$, the absolute and full-scale sensitivities are numerically the same. The measured value for $S_{\mathrm{A}}$ is $64 \mathrm{kHz} / \mathrm{N}$ and for $S_{\mathrm{FS}}$ it is $29 \mathrm{kHz} / \mathrm{N}$. The resolution of the force sensor will be determined by the sensitivity and the accuracy of measuring the resonance frequency.

\section{Conclusions}

A silicon resonant force sensor with piezoelectric excitation and detection was realized by using standard silicon processing, $\mathrm{ZnO}$ sputtering and bulk micromachining. If the thickness of the different layers in the multilayer structure is adjusted, it is possible to tailor the residual stress, resulting in a small off-set force on the sensor beam. The temperature effect due to mismatch of the thermal expansion coefficient will remain.

A piezoelectric layer of $\mathrm{ZnO}$ is used for the electrical excitation and detection of the vibration of the sensor beam. If an external load is applied, the resonance frequency of the sensor beam will shift. The measurements of the external force-to-frequency conversion agree well with the theory. This theory includes not only the force-to-frequency conversion but also the force conversion from the external load force to the sensor force. The performance of the sensor is characterized by the absolute and full-scale sensitivities. The measured values are $64 \mathrm{kHz} / \mathrm{N}$ for the absolute and $29 \mathrm{kHz} / \mathrm{N}$ for the full-scale sensitivity. 


\section{Acknowledgements}

The authors want to thank Siebe Bouwstra for discussions about the mechanical modelling, Peter Lenoir for building the measurement set-up and John Baxter for carefully reading the manuscript. This research in the program of the Dutch Foundation for Fundamental Research on Matter (FOM) is sponsored by the Netherlands Technology Foundation (STW).

\section{References}

1 J. G. Smits, H. A. C. Tilmans and T. S. J. Lammerink, Pressure dependence of resonant diaphragm pressure sensor, Proc. 3rd Int. Conf. Solid-State Sensors and Actuators (Transducers '85), Philadelphia, P.A, U.S.A., June 11-14, 1985, pp. 93-96.
2 H. A. C. Tilmans, S. Bouwstra, J. H. J. Fluitman and S. L. Spence, Design considerations for micromechanical sensors using encapsulated built-in resonant strain gauges, Sensors and Actuators A, 25-27(1991) 79-86.

3 D. L. Polla and R. S. Muller, Zinc oxide thin films for integrated sensor applications, Tech. Digest, IEEE Solid-State Sensors Workshop, Hilton Head Island, SC, U.S.A., June 6-9, 1986.

4 F. R. Blom, D. J. Yntema, F. C. M. van de Pol, M. Elwenspoek, J. H. J. Fluitman and Th. J. A. Popma, Thin-film $\mathrm{ZnO}$ as micromechanical actuator at low frequencies, Sensors and Actuators, A21-A23 (1990) 226-228.

5 F. R. Blom, F. C. M. van de Pol, G. Bauhuis and Th. J. A. Popma, (R.F. planar magnetron sputtered) $\mathrm{ZnO}$ films as piezoelectric transducers at low frequencies (part II), Thin Solid Films, submitted for publication.

6 F. R. Blom, S. Bouwstra, J. H. J. Fluitman and M. Elwenspoek, Resonating silicon beam force sensor, Sensors and Actuators, 17 (1989) 513-519.

7 K. D. Wise, Integrated sensors: interfacing electronics to a non-electronic world, Sensors and Actuators, 2 (1982) 229-237. 